\title{
Understanding Reduced Gravity Effects on Early Plant Development Before Attempting Life-Support Farming in the Moon and Mars
}

\author{
F. Javier Medina *, Aránzazu Manzano, Alicia Villacampa, Malgorzata Ciska and Raúl Herranz \\ Centro de Investigaciones Biológicas Margarita Salas-CSIC, Madrid, Spain
}

Plants are a necessary component of any system of bioregenerative life-support for human space exploration. For this purpose, plants must be capable of surviving and adapting to gravity levels different from the Earth gravity, namely microgravity, as it exists on board of spacecrafts orbiting the Earth, and partial-g, as it exists on the surface of the Moon or Mars. Gravity is a fundamental environmental factor for driving plant growth and development

OPEN ACCESS

Edited by:

Cyprien Verseux,

University of Bremen, Germany

Reviewed by:

Svetlana Poddubko,

Institute of Biomedical Problems

(RAS), Russia

Lucie Poulet,

NASA Postdoctoral Program,

United States

*Correspondence:

F. Javier Medina

fjmedina@cib.csic.es

Specialty section:

This article was submitted to

Astrobiology,

a section of the journal

Frontiers in Astronomy and Space

Sciences

Received: 22 June 2021

Accepted: 19 August 2021

Published: 03 September 2021

Citation:

Medina FJ, Manzano A, Villacampa A, Ciska M and Herranz R (2021)

Understanding Reduced Gravity

Effects on Early Plant Development

Before Attempting Life-Support

Farming in the Moon and Mars.

Front. Astron. Space Sci. 8:729154.

doi: $10.3389 /$ fspas.2021.729154 through gravitropism. Exposure to real or simulated microgravity produces a stress response in plants, which show cellular alterations and gene expression reprogramming. Partial-g studies have been performed in the ISS using centrifuges and in ground based facilities, by implementing adaptations in them. Seedlings and cell cultures were used in these studies. The Mars gravity level is capable of stimulating the gravitropic response of the roots and preserving the auxin polar transport. Furthermore, whereas Moon gravity produces alterations comparable, or even stronger than microgravity, the intensity of the alterations found at Mars gravity was milder. An adaptive response has been found in these experiments, showing upregulation of WRKY transcription factors involved in acclimation. This knowledge must be improved by incorporating plants to the coming projects of Moon exploration.

Keywords: root meristem, cell proliferation, ribosome biogenesis, auxin, transcriptomics, random positioning machine, International Space Station, space farming

\section{INTRODUCTION}

The achievement of plant cultivation in space, also called "space farming" is an important step in the development of bioregenerative life-support systems (BLSS) to enable long-term human space exploration. Plants are fundamental elements of these BLSS, since they can provide unique and essential factors and components for the support of the human life in extra-terrestrial environments. They are a source of oxygen and supply a variety of valuable nutrients of different types, including vitamins; they contribute to the regulation of the atmospheric humidity and are capable of removing and recycling carbon dioxide, one of the major waste elements of life whose accumulation destabilizes environmental balances (Ferl et al., 2002; Wheeler, 2017). Last, but not least, plant culture is an activity recognized by astronauts and cosmonauts as highly rewarding in terms of psychological well-being, a non-negligible factor to be taken into account for the success of space exploration enterprises (Haeuplik-Meusburger et al., 2014; Zhang et al., 2020).

The first step of space exploration consists of leaving the Earth, stepping into the outer reaches of atmosphere. Space explorers, and their accompanying plants, are exposed to microgravity 
conditions, as they exist on spacecrafts and stations orbiting the Earth, such as the International Space Station (ISS). Moreover, to enable human settlements on nearby planets, the influence on the plant physiology of partial or reduced gravity levels, such as those existing on the surface of the Moon $(1 / 6=0.17 \mathrm{~g})$ or Mars $(3 / 8=$ $0.38 \mathrm{~g}$ ) should be investigated (Kiss, 2014). Plant response to these partial gravity levels, is of utmost importance taking into account the plans of the space agencies to travel back to the Moon (Artemis and Gateway) in 2024 (Chavers et al., 2019; Von Ehrenfried, 2020) and to Mars in the near future. In this respect, it is worth mentioning that a terrestrial cotton seed was reported to have germinated on the lunar surface on board of the Chinese Chang'e 4 probe, which landed on the Moon in January 2019. Seed germination took place within the "life-regeneration ecosystem" facility carried by the craft. Details of the experiment, following the life trajectory of cotton seed germination, development, and final fate after long term exposure to super cold temperature, are publicly available, although only in the form of a preprint, and not of a peer-reviewed publication (Xie et al., 2021).

\section{PLANTS AND GRAVITY. PLANT RESPONSE AND ACCLIMATION TO MICROGRAVITY}

Plants of different species have been successfully grown in space, under microgravity conditions, on numerous occasions, reaching different developmental stages, including the completion of the full seed-to-seed life cycle (Merkys et al., 1984; Musgrave et al., 2000; Karahara et al., 2020). The earliest plant growth experiment was performed within the Oasis 1 hardware aboard Salyut 1 in 1971 (Harvey and Zakutnyaya, 2011; reviewed by Zabel et al., 2016). Then, experiments were performed on board of spacecrafts such as the NASA Space Shuttle or the Soviet MIR Station, but the assembly and operation of the International Space Station (ISS) was a giant step forward in the achievement of this objective (Vandenbrink and Kiss, 2016). In parallel, similar plant growth experiments on Earth, using ground based facilities for microgravity simulation, such as clinostats and random positioning machines have been successfully run (Kiss et al., 2019). They have provided a reliable replica of the biological results obtained in space experiments under real microgravity, even though the gravity vector is not possible to be avoided or removed on the Earth surface (Herranz et al., 2013; Medina et al., 2015; Van Loon, 2016).

These works using plants should be considered in the context of studies of microgravity effects extensively carried out in a wide range of living organisms, including the commonly recognized biological model species of most taxonomical categories, from mammals to bacteria. In general, microgravity is perceived by living beings as a stress-generating environmental factor, although the intensity of the physiological alterations caused is rather variable between the different organisms (Bizzarri et al., 2015; Van Loon et al., 2020). Two effects of the altered or null gravity signal, one direct and another indirect, overlap in the generation of a biological response: the direct effect is the modification of physiological processes (e.g., the changes in

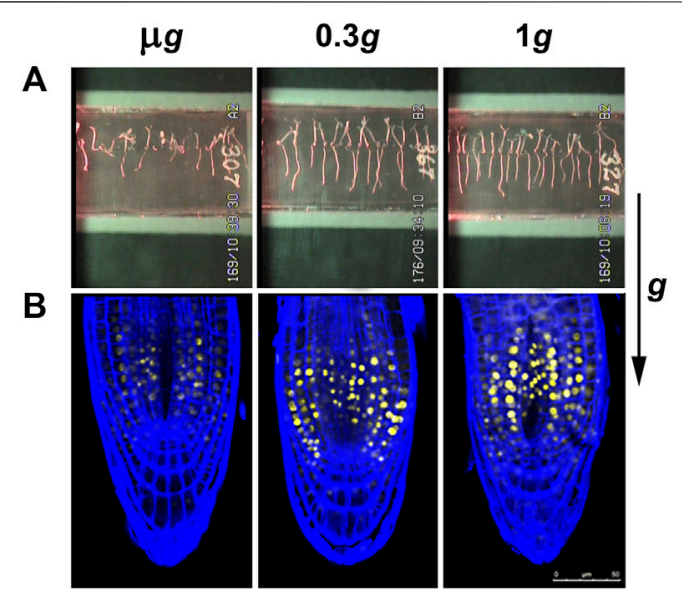

FIGURE 1 | Images obtained from the spaceflight experiment "Seedling Growth" at different levels of gravity. Seedlings of Arabidopsis thaliana were grown in the ISS for a period of 6 days from seed hydration, within the EMCS facility. Seedlings grew under a white light photoperiod for 4 days and under lateral red light photoactivation for the last 2 days. The gravity conditions were constant throughout the entire growth period. The levels $0.3 \mathrm{~g}$ (approx. the Mars gravity) and $1 \mathrm{~g}$ (Earth gravity) were obtained with a built-in centrifuge. (A): Images of seedlings in growth chambers (cassettes) at the end of the growth period. Seedlings grown in microgravity $(\mu \mathrm{g})$ appear disoriented, whereas under 0.3 and $1 \mathrm{~g}$ seedlings show a conspicuous gravitropism (the direction of the $g$ vector is indicated at the right). (B): Confocal microscopic images of the root tips of seedlings of the DII-Venus reporter line of $A$. thaliana grown in the same conditions. Yellow fluorescence corresponds to a negative pattern of the distribution of the phytohormone auxin. Roots were counterstained with the SCRI 2200 dye (Renaissance Chemicals, North Duffield, UK) for the visualization of the cell wall in blue. Roots grown in microgravity show an altered pattern of auxin distribution with respect to the $1 \mathrm{~g}$ control, indicative of some inhibition of the auxin polar transport. On the contrary, the pattern of roots grown at $0.3 \mathrm{~g}$ is basically similar to the control pattern, indicating that auxin polar transport is not altered at these partial-g levels.

the mechanisms of biochemical reactions); the indirect effect is the physical change of collateral elements or factors which are not intrinsic part of the physiological processes, but may have an influence on them. Examples of these side-effects are the diffusion of water in the soil, that may alter the correct hydration of roots, or the convective processes, that may influence the correct gas exchange. It is rather difficult to separate these two types of effects when analyzing experimental results, although some attempts have been done using simulated microgravity (Herranz et al., 2013). These indirect effects can be mitigated with adequate ventilation and adequate watering systems, as is currently being done in Veggie and APH facilities on the ISS (Massa et al., 2016; Monje et al., 2019; Monje et al., 2020).

Moreover, these effects are difficult to overcome since terrestrial organisms have evolved under a constant gravity vector (Bizzarri et al., 2015). Although, in principle, terrestrial organisms do not seem prepared to be confronted with an environment devoid of the gravity force, and they indeed lack of a genetic equipment specialized in the response to this condition, signs of acclimation and adaptation processes have been found in some species, as a result of their genetic plasticity, a feature greatly varying between different taxa (Paul et al., 2012; 
Medina et al., 2021). Actually, comprehensive studies on these processes are still insufficient and it appears that, similar as the intensity of the gravitational stress, different taxonomic categories display a different adaptability.

In the particular case of higher plants, gravity is an environmental factor decisively affecting plant growth, by means of the process called gravitropism, which modulates the growth orientation according to the gravity vector, with positive root gravitropism and negative shoot gravitropism. Nevertheless, in microgravity, the cue for this tropism (i.e., the gravity vector) is not present. The consequence is the substitution of gravitropism by automorphogenesis, a process producing in roots spontaneous curvatures followed by straight root elongations in random directions (Hoson and Soga, 2003; Driss-Ecole et al., 2008). This is accompanied by an intense reorganization of the transport and distribution of phytohormones, mostly affecting auxin, but also cytokinin, according to the available data, still incomplete, obtained in real and simulated microgravity (Manzano et al., 2013; Ferl and Paul, 2016; Yamazaki et al., 2016) (Figure 1). This hormonal perturbation is transduced to meristem, producing the alteration of cell proliferation rate and ribosome biogenesis during seedling development (Matía et al., 2010), which could have consequences at the level of the developmental pattern of the plant, ultimately relying on meristematic activity. In addition, an observed effect of microgravity is the induction in the plant of a higher sensitivity to other environmental cues, such as moisture or light, that are usually masked by dominant gravitropism (Manzano et al., 2021).

Physiological and cellular alterations are related to changes in gene expression, which have been identified by transcriptomic studies from space and ground experiments (Paul et al., 2012; Kwon et al., 2015; Johnson et al., 2017; Paul et al., 2017; Choi et al., 2019; Herranz et al., 2019; Kamal et al., 2019; Vandenbrink et al., 2019; Kruse et al., 2020; Villacampa et al., 2021a). In some cases, complementary proteomic studies were also performed (Kruse et al., 2020). Other than real or simulated microgravity, the environmental conditions of these experiments were far from homogeneous, which complicated direct comparisons. To overcome this inconvenience, an important effort of data sharing and harmonizing has been undertaken in the NASAled initiative called GeneLab (Ray et al., 2018). This effort has allowed the identification of some common responses, often related to general mechanisms of defense against stress, such as the system of heat shock genes producing Heat Shock Proteins (HSP), which are molecular chaperones acting to protect and refold proteins in response to cellular damage. In addition, the oxidative stress pathways, involving the production of Reactive Oxygen Species (ROS), the cell wall remodeling system, cytoskeleton alterations and plastid genes, including those regulating photosynthesis, were included in the core set of functions appearing affected by spaceflight environment (Correll et al., 2013; Zupanska et al., 2013; Kwon et al., 2015; Johnson et al., 2017; Choi et al., 2019; Kruse et al., 2020). In none of these transcriptomic studies, specific genes for the response of plants to microgravity, or to the spaceflight environment, were found. For some authors these differentially expressed genes
(DEGs) represent alterations caused by the gravitational stress (Johnson et al., 2017; Choi et al., 2019), whereas other interpretations highlight the fact that DEGs were found from viable samples, indicating that they reflect the mechanism of physiological adaptation of plants to spaceflight (Paul et al., 2012; Paul et al., 2017).

In any case, whether due to or despite genetic and physiological alterations, plants are capable of acclimating to the environmental change, since they survive and fulfill their entire life cycle in the microgravity environment of spaceflight (De Micco et al., 2014; Karahara et al., 2020; Khodadad et al., 2020). Most likely, plants could trigger an early and primary acclimation response to the environmental change from Earth to space, to overcome the early alterations that have been repeatedly detected in seedlings. In more advanced developmental stages, more stable adaptive responses could be produced, including genetic and epigenetic changes capable of being transmitted to the offspring, allowing the survival of plants in the space environment throughout successive generations. Many uncertainties on these mechanisms still remain and they pose a fundamental challenge for space plant biology, now and for the coming years.

\section{GROWTH OF PLANTS IN FRACTIONAL GRAVITY. STUDIES IN SPACE AND IN GROUND BASED FACILITIES}

In contrast with the body of research acquired dealing with plant growth and development in microgravity, the number of studies investigating plant response to fractional or partial levels of $g$ is considerably reduced. Prior to the assembly and operation of ISS only a few studies were carried out using partial- $g$ levels, with the specific interest of the determination of thresholds for gravity detection (Kiss, 2014). On board of the American shuttle Columbia, using a centrifuge microscope, the orientation of the unicellular motile algae Euglena in water at different levels of gravity (gravitaxis) was followed. The threshold capable of triggering a gravitactic response was determined to be around $0.16 \mathrm{~g}$ (Häder et al., 1996). In other experiment, the gravitropic statolith system of the rhizoids of the green alga Chara was investigated in sounding rockets and also in parabolic flights using an on-board centrifuge. Acceleration of $0.14 g$ resulted in a displacement of statoliths toward the cell wall in the periphery of the cells, while acceleration of $0.05 \mathrm{~g}$ did not result in statolith movement (Limbach et al., 2005).

Once the ISS was assembled, the European Modular Cultivation System (EMCS), which was installed and actively operated from 2008 to 2018, made possible a significant progress in our knowledge of the plant response to spaceflight conditions by offering a reliable and versatile control of different environmental parameters related to plant growth, by overcoming some of the aforementioned unwanted side-effects of the microgravity environment, such as those affecting convection and watering. In addition, for the specific case of studies at fractional gravity levels, it provided the ability to apply different $g$-forces in space by means of a built-in centrifuge 
(Brinckmann, 2005; Kittang et al., 2014). An intelligent and effective policy of cooperative agreements between agencies regarding the use of this facility multiplied the outcomes of the experiments performed in it. As an outstanding example, the NASA-ESA "Seedling Growth" series of experiments was executed in this hardware to test the influence of blue and red light photostimulation in the plant response to the reduced gravity stimuli (Kiss et al., 2014). In a first experiment, different gravity levels were applied (microgravity; $0.1 \mathrm{~g}$; Moon; Mars; near-Earth $g$-level; $1 \mathrm{~g}$ ) to blue-light stimulated Arabidopsis thaliana seedlings grown for 6 days and demonstrated a replacement of gravitropism by blue-light-based phototropism signaling at microgravity level (Vandenbrink et al., 2019), but a striking stress response was found at $0.1 \mathrm{~g}$. Different components of the transcriptional response to the lack of gravity were determined as the $g$-gradient was progressively reduced (Herranz et al., 2019). In a successive experiment, three $g$ levels (microgravity, Mars gravity level and $1 g$ ground reference run) were applied and photostimulation was with red light. In this case, the analysis was carried out by combining morphological and molecular approaches (confocal and electron microscopy, and transcriptomics). Fundamental novel findings were obtained from this experiment. Firstly, seedlings grown under Mars gravity exhibited a conspicuous root gravitropic response, and, consequently, a balanced distribution of the phytohormone auxin throughout the root, indicative of a regular auxin polar transport (Figure 1). Furthermore, microgravity and partial gravity were found to trigger differential responses. The microgravity environment activates hormonal routes responsible for cell proliferation and cell growth and upregulates plastid/mitochondrial-encoded transcript expression, even in the dark. In contrast, the Mars gravity level inhibits these routes and activates responses to stress factors to restore cell growth parameters, only when red photostimulation is provided. This response is accompanied by upregulation of several transcription factors (TFs), such as the environmental acclimation-related WRKY family (Villacampa et al., 2021a). WRKYs are a family of numerous TFs, many of them involved in both abiotic and biotic stress responses, related to hormonal signaling and in acclimation processes (Phukan et al., 2016). This is a strong indication of the triggering of a transcriptional adaptive response to the new environment. Further validation of all transcriptomic results was obtained by comparison to other transcriptomic data obtained from $A$. thaliana seedlings grown during spaceflights and available in the GeneLab database (Ray et al., 2018).

Other studies in ISS have looked further into the determination of the threshold of gravity perceived by roots. A $g$-value of $2.0 \times 10^{-3} \mathrm{~g}$, estimated punctually at $1.4 \times 10^{-5} \mathrm{~g}$, was found enough to trigger some motion in statoliths, although the participation of the actomyosin system in this motion was considered essential (Driss-Ecole et al., 2008).

In parallel to the works carried out in space facilities, some studies have used terrestrial devices to reproduce Moon or Mars gravity levels and study their biological effects in experiments performed on Earth. An outstanding case of such these experiments consisted of the use of the Random Positioning
Machine (RPM) and the incubation of A. thaliana seedlings in this device for the biological validation of the terrestrial analog of the partial- $g$ conditions (Manzano et al., 2018). The RPM was originally designed to simulate microgravity, but an adaptation of this device was developed to expose living samples to simulated fractional levels of gravity. For this purpose, two different paradigms were used, one by implementing a centrifuge on a nominally operating RPM $\left(\mathrm{RPM}^{\mathrm{HW}}\right)$, and the other by applying specific software protocols to driving the RPM motors, thus changing the nominal mode of operation of the facility $\left(\mathrm{RPM}^{\mathrm{SW}}\right)$. Both approaches produced similar results, but the RPM software version showed an ideal working range at 0.05-0.4 $g$, while the RPM hardware version may work better at gravity levels above $0.3 \mathrm{~g}$. The effects of the simulated partial gravity were tested in plant root meristematic cells, a system with known response to real and simulated microgravity. Cell proliferation appeared increased and cell growth was reduced under Moon gravity, compared with the $1 g$ control. The difference with control was even higher at the Moon $g$ level than in simulated microgravity, indicating that meristematic competence (balance between cell growth and proliferation) was also affected at this gravity level. However, the results at the simulated Mars $g$-level were close to the $1 g$ static control. This suggests that we can separate the levels of gravity capable of being sensed at a molecular level by different intracellular mechanisms, from the gravity threshold capable of triggering a response in the root, visually detectable in the form of a defined growth direction according to the gravity vector. This threshold requires a level intermediate between Moon and Mars gravity (Manzano et al., 2018).

The RPM ${ }^{\mathrm{HW}}$ system was also used to incubate in vitro cell cultures of A. thaliana for a detailed study of the effects of simulated Mars gravity on the regulation of cell cycle progression and cell growth. Different times of exposure were tested. The effects observed at the Mars gravity levels were qualitatively similar as those caused by microgravity, but quantitatively less intense. Alteration in cell cycle regulator mechanisms associated to changes in the levels of some selected factors was detected, leading to changes in the duration of cell cycle phases, according to flow cytometry data. Nucleolar activity for ribosome biogenesis was reduced progressively with the exposure time and epigenetic mechanisms involved in chromatin remodeling were also altered (Kamal et al., 2018). Cell cultures were also exposed to partial- $g$ levels by using parabolic flights and a clinostat (Fengler et al., 2016). It is worth noting that the reliability of the results obtained with these procedures is questionable. In the case of the parabolic flight, the alternation of the exposure of the biological samples to different levels of gravity in the same parabola complicates the attribution of specific effects to defined causes; in the case of the clinostat, fast clinorotation has been shown to produce unwanted side effects and additional forces that complicate the determination of the actual g-force sensed by the biological sample (Villacampa et al., 2021b; Herranz et al., 2021). Transcriptomic data obtained from the exposed cell cultures show a lower number of DEGs than in the case of microgravity and an 
enrichment of DEGs in the cell wall metabolism and remodeling category (Fengler et al., 2016).

An interesting alternative for the study of the response of living beings, and particularly plants, to hypogravity is to make use of the Reduced Gravity Paradigm (RGP). It is based on the premise that adaptations expected or seen going from a higher to a lower gravity level are similar as changes seen going from $1 g$ to microgravity or partial gravity (Van Loon, 2016). The paradigm is not focused on the absolute acceleration values, but rather on the responses generated due to the delta between two gravity levels and it can be put in practice by using centrifuges for incubation of plant samples. In a typical experiment, the plant sample is incubated at the $2 g$ level until it shows a stable, steady state physiology. Then the acceleration of gravity is reduced and the plant will respond to this reduction. The hypothesis is that the adaptation e.g., from 2 to $1 \mathrm{~g}$ is similar to the adaptation from $1 \mathrm{~g}$ to microgravity. The magnitudes of the responses might be different, but the directions of the response would be the same (Van Loon, 2016).

\section{FUTURE PROSPECTS}

In the long term, studies on plant growth and development at fractional $g$-levels will pave the way to understand the molecular mechanisms to improve the cultivation conditions of plants on different planets. These discoveries can be applied in the design of bioregenerative life-support systems and space farming. Regarding the use of ground based facilities, further research is needed, especially around and beyond Mars $g$-levels, to better understanding the differences and constraints in the use of these facilities for the space biology community. However, the most important effort should be invested in incorporating plants to the coming initiatives of deep space exploration. The cis-lunar space considered for the Gateway project should be efficiently used for incorporating plants (both model and crop species) to a research platform that will be a fundamental tool to fill this gap in knowledge (Paul and Ferl, 2018). This will be crucial for further exploration initiatives, such as an extended mission to Mars.

\section{REFERENCES}

Bizzarri, M., Monici, M., and Loon, J. J. W. A. v. (2015). How Microgravity Affects the Biology of Living Systems. Biomed. Res. Int. 2015, 863075. doi:10.1155/ 2015/863075

Brinckmann, E. (2005). ESA Hardware for Plant Research on the International Space Station. Adv. Space Res. 36, 1162-1166. doi:10.1016/j.asr.2005.02.019

Chavers, G., Suzukib, N., Smithc, M., Watson-Morgan, L., Clarke, S. W., Engelund, W. C., Aitchison, L., Mceniry, S., Means, L., and Deklotz, M. (2019). "NASA'S Human Lunar Landing Strategy," in 70th International Astronautical Congress 2019, Washington DC, USA. Paper ID: 54732.

Choi, W.-G., Barker, R. J., Kim, S.-H., Swanson, S. J., and Gilroy, S. (2019). Variation in the Transcriptome of Different Ecotypes of Arabidopsis Thalianareveals Signatures of Oxidative Stress in Plant Responses to Spaceflight. Am. J. Bot. 106, 123-136. doi:10.1002/ajb2.1223

Correll, M. J., Pyle, T. P., Millar, K. D. L., Sun, Y., Yao, J., Edelmann, R. E., et al. (2013). Transcriptome Analyses of Arabidopsis thaliana Seedlings Grown in

\section{DATA AVAILABILITY STATEMENT}

The original contributions presented in the study are included in the article/Supplementary Material, further inquiries can be directed to the corresponding author.

\section{AUTHOR CONTRIBUTIONS}

FJM received the invitation to contribute to the Research Topic, proposed the topic to the rest of authors and wrote the first draft of the manuscript. AM, AV, MC, and $\mathrm{RH}$ carried out the experimental works reported in the manuscript, actively discussed the topics, ideas, concepts and hypothesis contained in the manuscript, provided corrections to the draft manuscript and approved the final version.

\section{FUNDING}

Work performed in the authors' laboratory was supported by different grants of the Spanish National Agency for Research of the Spanish Government, e.g., Grants \#ESP2015-64323-R and \#RTI2018-099309-B-I00 (co-funded by EU-ERDF). Access to ISS was made possible by ESA and NASA, and the use of groundbased facilities for microgravity simulation was supported by the ESA-CORA-GBF Program.

\section{ACKNOWLEDGMENTS}

We wish to thank Prof. John Z. Kiss (University of North Carolina at Greensboro, United States), and Dr. Ing. Jack van Loon (ESA-ESTEC and Free University of Amsterdam, Netherlands) for the continuous and fruitful exchange of data, results and ideas in relation to the topics covered in this paper. We would also like to express our gratitude to astronauts, technicians, engineers, and management personnel who has participated in our spaceflight and ground-based experiments.

Space: Implications for Gravity-Responsive Genes. Planta 238, 519-533. doi:10.1007/s00425-013-1909-x

De Micco, V., De Pascale, S., Paradiso, R., and Aronne, G. (2014). Microgravity Effects on Different Stages of Higher Plant Life Cycle and Completion of Theseed-To-Seedcycle. Plant Biol. J. 16, 31-38. doi:10.1111/plb.12098

Driss-Ecole, D., Legué, V., Carnero-Diaz, E., and Perbal, G. (2008). Gravisensitivity and Automorphogenesis of Lentil Seedling Roots Grown on Board the International Space Station. Physiol. Plantarum 134, 191-201. doi:10.1111/ j.1399-3054.2008.01121.x

Fengler, S., Spirer, I., Neef, M., Ecke, M., Hauslage, J., and Hampp, R. (2016). Changes in Gene Expression of Arabidopsis Thaliana Cell Cultures Upon Exposure to Real and Simulated Partial-G Forces. Microgravity Sci. Technol. 28, 319-329. doi:10.1007/s12217-015-9452-y

Ferl, R. J., and Paul, A.-L. (2016). The Effect of Spaceflight on the Gravity-Sensing Auxin Gradient of Roots: GFP Reporter Gene Microscopy on Orbit. npj Microgravity 2, 15023. doi:10.1038/npjmgrav.2015.23

Ferl, R., Wheeler, R., Levine, H. G., and Paul, A.-L. (2002). Plants in Space. Curr. Opin. Plant Biol. 5, 258-263. doi:10.1016/S1369-5266(02)00254-6 
Häder, D.-P., Rosum, A., Schäfer, J., and Hemmersbach, R. (1996). Graviperception in the Flagellate Euglena Gracilis during a Shuttle Space Flight. J. Biotechnol. 47, 261-269. doi:10.1016/0168-1656(96)01374-0

Haeuplik-Meusburger, S., Paterson, C., Schubert, D., and Zabel, P. (2014). Greenhouses and Their Humanizing Synergies. Acta Astronautica 96, 138-150. doi:10.1016/j.actaastro.2013.11.031

Harvey, B., and Zakutnyaya, O. (2011). Russian Space Probes: Scientific Discoveries and Future Missions. Chichester UK: Springer science $\&$ business media/Praxis Publishing. doi:10.1007/978-1-4419-8159-9

Herranz, R., Anken, R., Boonstra, J., Braun, M., Christianen, P. C. M., De Geest, M., et al. (2013). Ground-Based Facilities for Simulation of Microgravity: Organism-Specific Recommendations for Their Use, and Recommended Terminology. Astrobiology 13, 1-17. doi:10.1089/ast.2012.0876

Herranz, R., Vandenbrink, J. P., Villacampa, A., Manzano, A., Poehlman, W. L., Feltus, F. A., et al. (2019). RNAseq Analysis of the Response of Arabidopsis thaliana to Fractional Gravity Under Blue-Light Stimulation During Spaceflight. Front. Plant Sci. 10, 1529. doi:10.3389/fpls.2019.01529

Herranz, R., Valbuena, M. A., Manzano, A., Kamal, K. Y., Villacampa, A., Ciska, M., et al. (2021). "Use of Reduced Gravity Simulators for Plant Biological Studies," in Plant Gravitropism: Methods and Protocols Methods in Molecular Biology. Editor E. Blancaflor 2nd Edition (New York, NY: Springer Science+Business Media, LLC, part of Springer Nature). doi:10.1007/978-10716-1677-2_16

Hoson, T., and Soga, K. (2003). New Aspects of Gravity Responses in Plant Cells. Int. Rev. Cytol. 229, 209-244. doi:10.1016/S0074-7696(03)29005-7

Johnson, C. M., Subramanian, A., Pattathil, S., Correll, M. J., and Kiss, J. Z. (2017). Comparative Transcriptomics Indicate Changes in Cell Wall Organization and Stress Response in Seedlings During Spaceflight. Am. J. Bot. 104, 1219-1231. doi:10.3732/ajb.1700079

Kamal, K. Y., Herranz, R., Van Loon, J. J. W. A., and Medina, F. J. (2018). Simulated Microgravity, Mars Gravity, and 2g Hypergravity Affect Cell Cycle Regulation, Ribosome Biogenesis, and Epigenetics in Arabidopsis Cell Cultures. Sci. Rep. 8, 6424. doi:10.1038/s41598-018-24942-7

Kamal, K. Y., Van Loon, J. J. W. A., Medina, F. J., and Herranz, R. (2019). Differential Transcriptional Profile through Cell Cycle Progression in Arabidopsis Cultures Under Simulated Microgravity. Genomics 111, 1956-1965. doi:10.1016/j.ygeno.2019.01.007

Karahara, I., Suto, T., Yamaguchi, T., Yashiro, U., Tamaoki, D., Okamoto, E., et al. (2020). Vegetative and Reproductive Growth of Arabidopsis Under Microgravity Conditions in Space. J. Plant Res. 133, 571-585. doi:10.1007/ s10265-020-01200-4

Khodadad, C. L. M., Hummerick, M. E., Spencer, L. E., Dixit, A. R., Richards, J. T., Romeyn, M. W., et al. (2020). Microbiological and Nutritional Analysis of Lettuce Crops Grown on the International Space Station. Front. Plant Sci. 11, 199. doi:10.3389/fpls.2020.00199

Kiss, J. Z., Aanes, G., Schiefloe, M., Coelho, L. H. F., Millar, K. D. L., and Edelmann, R. E. (2014). Changes in Operational Procedures to Improve Spaceflight Experiments in Plant Biology in the European Modular Cultivation System. Adv. Space Res. 53, 818-827. doi:10.1016/j.asr.2013.12.024

Kiss, J. Z., Wolverton, C., Wyatt, S. E., Hasenstein, K. H., and Van Loon, J. J. W. A. (2019). Comparison of Microgravity Analogs to Spaceflight in Studies of Plant Growth and Development. Front. Plant Sci. 10, 1577. doi:10.3389/ fpls.2019.01577

Kiss, J. Z. (2014). Plant Biology in Reduced Gravity on the Moon and Mars. Plant Biol. J. 16, 12-17. doi:10.1111/plb.12031

Kittang, A.-I., Iversen, T.-H., Fossum, K. R., Mazars, C., Carnero-Diaz, E., Boucheron-Dubuisson, E., et al. (2014). Exploration of Plant Growth and Development Using the European Modular Cultivation System Facility on the International Space Station. Plant Biol. J. 16, 528-538. doi:10.1111/plb.12132

Kruse, C. P. S., Meyers, A. D., Basu, P., Hutchinson, S., Luesse, D. R., and Wyatt, S. E. (2020). Spaceflight Induces Novel Regulatory Responses in Arabidopsis Seedling as Revealed by Combined Proteomic and Transcriptomic Analyses. BMC Plant Biol. 20, 237. doi:10.1186/s12870-020-02392-6

Kwon, T., Sparks, J. A., Nakashima, J., Allen, S. N., Tang, Y., and Blancaflor, E. B. (2015). Transcriptional Response of Arabidopsis Seedlings during Spaceflight Reveals Peroxidase and Cell wall Remodeling Genes Associated with Root Hair Development. Am. J. Bot. 102, 21-35. doi:10.3732/ajb.1400458
Limbach, C., Hauslage, J., Schafer, C., and Braun, M. (2005). How to Activate a Plant Gravireceptor. Early Mechanisms of Gravity Sensing Studied in Characean Rhizoids during Parabolic Flights. Plant Physiol. 139, 1030-1040. doi:10.1104/pp.105.068106

Manzano, A., Larkin, O. J., Dijkstra, C. E., Anthony, P., Davey, M. R., Eaves, L., et al. (2013). Meristematic Cell Proliferation and Ribosome Biogenesis Are Decoupled in Diamagnetically Levitated Arabidopsis Seedlings. BMC Plant Biol. 13, 124. doi:10.1186/1471-2229-13-124

Manzano, A., Herranz, R., Den Toom, L. A., Te Slaa, S., Borst, G., Visser, M., et al. (2018). Novel, Moon and Mars, Partial Gravity Simulation Paradigms and Their Effects on the Balance between Cell Growth and Cell Proliferation during Early Plant Development. npj Microgravity 4, 9. doi:10.1038/s41526-0180041-4

Manzano, A., Pereda-Loth, V., De Bures, A., Sáez-Vásquez, J., Herranz, R., and Medina, F. J. (2021). Light Signals Provide a Mechanism of Counteracting Alterations Caused by Simulated Microgravity in Proliferating Plant Cells. Am. J. Bot. 108(9), 2021 In press.

Massa, G. D., Wheeler, R. M., Morrow, R. C., and Levine, H. G. (2016). "Growth chambers on the International Space Station for Large Plants," in VIII International Symposium on Light in Horticulture, East Lansing MI, USA. doi:10.17660/actahortic.2016.1134.29

Matía, I., González-Camacho, F., Herranz, R., Kiss, J. Z., Gasset, G., Van Loon, J. J. W. A., et al. (2010). Plant Cell Proliferation and Growth are Altered by Microgravity Conditions in Spaceflight. J. Plant Physiol. 167, 184-193. doi:10.1016/j.jplph.2009.08.012

Beysens, D. A., van Loon, J. J. W., Arena, C., Aronne, G., and De Micco, V. (2015). "Generation and Applications of Extra-terrestrial Environments on Earth," in Generation and Applications of Extra-Terrestrial Environments on Earth. Editors D. Beysens and J.J.W.A. Van Loon (Delft, NL: River Publishers), 1-318. doi:10.13052/rp-9788793237544

Medina, F. J., Manzano, A., Kamal, K. Y., Ciska, M., and Herranz, R. (2021). "Plants in Space: Novel Physiological Challenges and Adaptation Mechanisms," in Progress in Botany. Editors U. Lüttge, F.M. Cánovas, H. Pretzsch, M.C. Risueño, and C. Leuschner (Berlin, Heidelberg: Springer Nature), 83, 1-36. doi:10.1007/ 124_2021_53

Merkys, A. J., Laurinavičius, R. S., and Švegždiene, D. V. (1984). Plant Growth, Development and Embryogenesis during Salyut-7 Flight. Adv. Space Res. 4, 55-63. doi:10.1016/0273-1177(84)90224-2

Monje, O., Richards, J. T., Hanson, D. T., Turpin, M. M., Moinuddin, S. G. A., Costa, M. A., Davin, L. B., Lewis, N. G., Carver, J. C., and Dimapilis, D. (2019). "New Perspectives for Watering Substrate-Based Root Modules in Microgravity in the Advanced Plant Habitat (APH)," in 35th Annual Meeting of the American Society for Gravitational and Space Research, Denver, CO; United States, 20 - 23 November 2019

Monje, O., Richards, J. T., Carver, J. A., Dimapilis, D. I., Levine, H. G., Dufour, N. F., et al. (2020). Hardware Validation of the Advanced Plant Habitat on ISS: Canopy Photosynthesis in Reduced Gravity. Front. Plant Sci. 11, 673. doi:10.3389/fpls.2020.00673

Musgrave, M. E., Kuang, A., Xiao, Y., Stout, S. C., Bingham, G. E., Briarty, L. G., et al. (2000). Gravity independence of Seed-To-Seed Cycling in Brassica Rapa. Planta 210, 400-406. doi:10.1007/PL00008148

Paul, A.-L., and Ferl, R. (2018). "Plants as Part of the Deep Space Exploration Schema," in Deep Space Gateway Concept Science Workshop, Denver CO, USA, 27-March 1, 2018. Contribution No. 2063, id.3059.

Paul, A.-L., Zupanska, A. K., Ostrow, D. T., Zhang, Y., Sun, Y., Li, J.-L., et al. (2012). Spaceflight Transcriptomes: Unique Responses to a Novel Environment Astrobiology 12, 40-56. doi:10.1089/ast.2011.0696

Paul, A.-L., Sng, N. J., Zupanska, A. K., Krishnamurthy, A., Schultz, E. R., and Ferl, R. J. (2017). Genetic Dissection of the Arabidopsis Spaceflight Transcriptome: Are Some Responses Dispensable for the Physiological Adaptation of Plants to Spaceflight?. PLoS One 12, e0180186. doi:10.1371/journal.pone.0180186

Phukan, U. J., Jeena, G. S., and Shukla, R. K. (2016). WRKY Transcription Factors: Molecular Regulation and Stress Responses in Plants. Front. Plant Sci. 7, 760. doi:10.3389/fpls.2016.00760

Ray, S., Gebre, S., Fogle, H., Berrios, D. C., Tran, P. B., Galazka, J. M., et al. (2018). GeneLab: Omics Database for Spaceflight Experiments. Bioinformatics 35, 1753-1759. doi:10.1093/bioinformatics/bty884 
Van Loon, J. J. W. A., Cras, P., Bouwens, W. H. A. C. M., Roozendaal, W., and Vernikos, J. (2020). Gravity Deprivation: Is it Ethical for Optimal Physiology?. Front. Physiol. 11, 470. doi:10.3389/fphys.2020.00470

Van Loon, J. J. W. A. (2016). Centrifuges for Microgravity Simulation. The Reduced Gravity Paradigm. Front. Astron. Space Sci. 3, 21. doi:10.3389/ fspas.2016.00021

Vandenbrink, J. P., and Kiss, J. Z. (2016). Space, the Final Frontier: A Critical Review of Recent Experiments Performed in Microgravity. Plant Sci. 243, 115-119. doi:10.1016/j.plantsci.2015.11.004

Vandenbrink, J. P., Herranz, R., Poehlman, W. L., Alex Feltus, F., Villacampa, A., Ciska, M., et al. (2019). RNA -seq Analyses of Arabidopsis thaliana Seedlings after Exposure to Blue-Light Phototropic Stimuli in Microgravity. Am. J. Bot. 106, 1466-1476. doi:10.1002/ajb2.1384

Villacampa, A., Ciska, M., Manzano, A., Vandenbrink, J. P., Kiss, J. Z., Herranz, R., et al. (2021a). From Spaceflight to Mars G-Levels: Adaptive Response of A. Thaliana Seedlings in a Reduced Gravity Environment Is Enhanced by RedLight Photostimulation. Int. J. Mol. Sci. 22, 899. doi:10.3390/ijms22020899

Villacampa, A., Sora, L., Herranz, R., Medina, F.-J., and Ciska, M. (2021b). Analysis of Graviresponse and Biological Effects of Vertical and Horizontal Clinorotation in Arabidopsis thaliana Root Tip. Plants 10, 734. doi:10.3390/ plants10040734

Von Ehrenfried, M. D. (2020). The Artemis Lunar Program: Returning People to the Moon. Cham: Springer Nature Switzerland AG. doi:10.1007/978-3-03038513-2

Wheeler, R. M. (2017). Agriculture for Space: People and Places Paving the Way. Open Agric. 2, 14-32. doi:10.1515/opag-2017-0002

Xie, G., Zhang, Y., Yang, J., Yu, D., Ren, M., Qiu, D., et al. (2021). Adaptation to Real 1/ 6 G Moon Gravity Contributes to Plant Development and Expeditious Acclimation to Super-Freezing. Res. Square - preprint. doi:10.21203/rs.3.rs-278675/v1

Yamazaki, C., Fujii, N., Miyazawa, Y., Kamada, M., Kasahara, H., Osada, I., et al. (2016). The Gravity-Induced Re-Localization of Auxin Efflux Carrier CsPIN1 in Cucumber Seedlings: Spaceflight Experiments for Immunohistochemical Microscopy. npj Microgravity 2, 16030. doi:10.1038/npjmgrav.2016.30

Zabel, P., Bamsey, M., Schubert, D., and Tajmar, M. (2016). Review and Analysis of over 40 Years of Space Plant Growth Systems. Life Sci. Space Res. 10, 1-16. doi:10.1016/j.lssr.2016.06.004

Zhang, W., Liu, H., Li, Z., and Liu, H. (2020). Synergistic Effects of Edible Plants with Light Environment on the Emotion and Sleep of Humans in LongDuration Isolated Environment. Life Sci. Space Res. 24, 42-49. doi:10.1016/ j.lssr.2019.11.003

Zupanska, A. K., Denison, F. C., Ferl, R. J., and Paul, A.-L. (2013). Spaceflight Engages Heat Shock Protein and Other Molecular Chaperone Genes in Tissue Culture Cells of Arabidopsis thaliana. Am. J. Bot. 100, 235-248. doi:10.3732/ ajb. 1200343

Conflict of Interest: The authors declare that the research was conducted in the absence of any commercial or financial relationships that could be construed as a potential conflict of interest.

Publisher's Note: All claims expressed in this article are solely those of the authors and do not necessarily represent those of their affiliated organizations, or those of the publisher, the editors and the reviewers. Any product that may be evaluated in this article, or claim that may be made by its manufacturer, is not guaranteed or endorsed by the publisher.

Copyright (c) 2021 Medina, Manzano, Villacampa, Ciska and Herranz. This is an open-access article distributed under the terms of the Creative Commons Attribution License (CC BY). The use, distribution or reproduction in other forums is permitted, provided the original author(s) and the copyright owner(s) are credited and that the original publication in this journal is cited, in accordance with accepted academic practice. No use, distribution or reproduction is permitted which does not comply with these terms. 